\title{
Analyzing the Landscape: Community Organizing and Health Equity
}

\author{
Jennifer J. García ${ }^{1}$, Cheryl Grills ${ }^{1}$, Sandra Villanueva1, Karren A. Lane ${ }^{2}$, Curtiss Takada-Rooks ${ }^{1}$, Christopher D. Hill ${ }^{3}$ \\ ${ }^{1}$ Psychology Applied Research Center, Loyola Marymount University, ${ }^{2}$ The Weingart Foundation, ${ }^{3}$ Bowie State University \\ Keywords: community based participatory research, health equity, community organizing, landscape analysis \\ https://doi.org/10.35844/001c.13196
}

Journal of Participatory Research Methods

Vol. 1, Issue 1, 2020

\begin{abstract}
In this paper we describe landscape analysis, a participatory research method for public health scholars interested in identifying and elucidating trends, opportunities, and gaps in the field. We used this method to understand the environmental and social conditions of primarily under-resourced communities of color, and identify key organizing strategies and practices used by community organizers to fight for policy and systems change around childhood health equity issues. Using a community-based participatory research approach, we developed and implemented a structured landscape analysis process among a national sample of 45 community-based organizations (CBOs). We discuss in detail our sampling procedures, protocol development, and analysis process. The resulting landscape analysis revealed similar challenges (e.g., lack of adequate housing, poor early childhood education resources) across diverse communities, and the best practices and innovative solutions used by $\mathrm{CBOs}$ to address these challenges. The landscape analysis process underscores the important role that social justice grassroots CBOs play in addressing the root causes of health inequity even though they may not identify, or be identified, as "public health" organizations.
\end{abstract}

\section{Analyzing the Landscape: Community Organizers and Health Equity}

Persistent disparities in health stem from social and structural conditions that disadvantage low-income communities of color and put the most vulnerable at increased risk for poor health outcomes (Larson et al., 2008). Health outcomes that disproportionately impact children of color, include overweight and obesity (Flores, 2010; Guerrero et al., 2016; Ogden et al., 2012), asthma (Flores, 2010), and birth outcomes (Blumenshine et al., 2010). Health disparities among children are particularly concerning as recent data suggest that for some indicators these disparities are widening (Mehta et al., 2013). Poor health in childhood contributes to increased healthcare costs across the lifespan, higher morbidity and mortality, and decreased quality of life (Braveman \& Barclay, 2009). Strategies and interventions seeking to reduce childhood health disparities must address the underlying social determinants including poverty, racism, and segregation (Braveman et al., 2011; Braveman \& Barclay, 2009; Minkler et al., 2019; Sanders-Phillips et al., 2009).

Community-based participatory research (CBPR) - a community-centered approach that strives to equitably involve community members and researchers as partners in the research process - has been used to garner community support for numerous collaborative projects focused on childhood health disparities (Israel et al., 2003, 2005). A CBPR approach leverages the combined expertise of all team members including the lived experience and insider knowledge that community members bring to the partnership. Israel and 
colleagues (2005) advocate for greater use of CBPR principles and strategies, especially when working with marginalized communities that have been historically excluded from the research process.

Community organizing philosophy dovetails with public health frameworks that prioritize root causes, social justice, and systems change (Minkler et al., 2019; Pastor et al., 2018) and a CBPR approach that centers community wisdom (Israel et al., 1998, 2003). A goal of community organizing is to support community empowerment, collective action, and advocacy to change the structures and systems that perpetuate inequity (Christens \& Speer, 2015). Community organizers accomplish this by directly engaging local residents most impacted by social injustice, with the aim of building and sustaining community power in order to advocate for systems change (Fisher et al., 2018; Grills et al., 2014; Minkler et al., 2019; Pastor et al., 2018; Wallerstein \& Duran, 2010). Organizers understand that social change and the fight for health equity requires a transformational, movement-building approach that transcends individual issues or campaigns (Pastor et al., 2011).

Community organizing in the context of public health has been defined as "grassroots movements that empower and mobilize individuals to act in their own collective self-interest to address community health problems by altering the balance of power, resource distribution, and policy decision making in their environments" (Subica et al., 2016, p. 80) and is gaining traction as a public health strategy to address the social determinants of health (Grills et al., 2014; Minkler et al., 2019; Pastor et al., 2018). For example, community organizing has been used to connect individuals to collective efforts that target community conditions harmful to health, including food and recreation environments (Grills et al., 2014; Subica et al., 2016), public safety (Speer et al., 2003), education (Gutiérrez \& Lewis, 2012), and environmental health (Cohen et al., 2016).

Grassroots social justice organizations, community organizers, and community members are key players in the fight for health equity. Organizers have direct knowledge of and experience with a range of social and structural issues that impact health and a deep understanding of community strengths and needs (Minkler et al., 2019). However, community organizing is decentralized and context-specific, making it difficult, at times, to track promising practices or innovative solutions in the field and share knowledge or resources across communities or content areas. To address these challenges, we sought a community-engaged, participatory research method to learn from community organizers about their work on the ground that would also support a big picture analysis to identify shared struggles and best practices in the field. We adapted a process commonly used in community organizing, referred to as landscape analysis, to understand the landscape of issues related to childhood health disparities that could inform community-driven strategies to address health inequities. Our landscape analysis process documents the social justice work and community organizing strategies used to support childhood health in resource-poor communities across the country. 
Specifically, the landscape analysis revealed important insights about the social and environmental conditions affecting childhood health and the strategies and practices that were most effective for supporting health equity. In this paper, we describe the CBPR framework and methods used in the landscape analysis process including our sampling strategy, development of tools, data collection, data analysis, and data dissemination. We discuss the benefits, limitations, and implications of this approach.

\section{Overview of the Landscape Analysis Process}

Field scanning (also called landscape scanning) is a practice used in philanthropy to identify the assets, needs, and gaps in a particular field (GrantCraft, 2012; Learning for Action, 2017; Sherry, 2013). Scanning takes a broad view to better understand opportunities, emerging issues, and field trends. For example, a field scan can help a funder to determine the best investment opportunities, thereby increasing efficiency, illuminate existing tools and strategies to avoid "reinventing the wheel," and identify opportunities for collaboration and relationship building (Sherry, 2013). This general approach has been applied to childhood mental health and education systems (Grife \& Werner, 2018; (Learning for Action, 2017). A similar method from the business world called environmental scans draws on both internal (e.g., organizational documents) and external information (e.g., social and political context) to inform strategic planning and assist with decision making (Graham et al., 2008). Environmental scans have been used by public health researchers and practitioners to design health programs and interventions (e.g., vaccination programs, cancer screenings) (Graham et al., 2008; Rowel et al., 2005; Wilburn et al., 2016).

The process itself varies depending on the purpose and goal of the scan, but methods include interviews with key constituents, surveys, literature and documents reviews, focus groups, visual mapping, or some combination of these methods (Sherry, 2013). Importantly, the information gleaned from the scan is actionable. While field scans and environmental scans are valuable tools for big picture assessment and planning, neither explicitly employs participatory methods, which is central to our work with marginalized communities and community organizers. Therefore, we adapted these approaches for use within a CBPR framework that utilizes participatory methods and a consistent social justice lens to analyze the landscape of health equity community organizing. We define landscape analysis as a participatory data collection and assessment process useful for understanding the broader context, evaluating strengths and challenges, and identifying field trends to inform actionable next steps. We use the term landscape analysis, rather than field scanning, to reflect the preferred terminology among the community organizing practitioners that we work with.

Our goal for the landscape analysis process was twofold. First, we wanted to produce a bird's eye analysis of community organizing efforts, based on what we learned from community organizers, about the most pressing issues facing their communities as well as the strategies and practices they employ to 
advance health equity. By capturing these stories from different communities across the country, our intent was to uplift localized efforts, identify promising or innovative solutions, and track trends and patterns on a national scale. The landscape analysis can serve as a resource for organizers, practitioners, and researchers working in the areas of grassroots social justice movements, health equity, and childhood health. Second, we wanted to engage in praxis by putting the research findings into action. Community organizers use landscape analysis to deepen their understanding of the scope, scale, and structural causes of the social and economic conditions they seek to address. An analysis of the landscape also informs an analysis of power - who benefits from the problem, who loses, how the organized power works toward the agenda, and how the organized power works against the agenda (Castellanos \& Pateriya, 2003). The landscape analysis is the first step in developing systems change campaigns and organizing strategies. Therefore, landscape analysis can be leveraged to 1) facilitate networking and alliance building and strengthen the movement by connecting organizations (i.e., by geographic region or by social justice issue area) that might otherwise not know about each other; 2) support sharedlearning, collaboration, and maximize the use of existing resources to support collaborative efforts, which can be significant for community-based organizations (CBOs) that are typically operating with limited resources; and 3) track trends and identify best practices for effective organizing and advocacy strategies across the field. In the following sections, we describe the application of the landscape analysis process to a community organizing health equity project, including details of the procedures and methods used, and discuss strengths and limitations of this approach.

\section{Communities Creating Healthy Environments}

Since its inception in 2009, the Communities Creating Healthy Environments (CCHE) initiative, funded by the Robert Wood Johnson Foundation (RWJF), recognized grassroots social justice organizing as a strategy to address childhood health injustice in marginalized communities using a CBPR framework (Grills et al., 2014). CCHE supports the work of grassroots social justice $\mathrm{CBOs}$ to advance environmental change using the art and science of community organizing. Over the past decade, the Praxis Project (Praxis) has served as the national program office and the Psychology Applied Research Center at Loyola Marymount University (PARC@LMU) as the national evaluator for CCHE. During Phases 1 (2009-2013) and 2 (2014-2016) of CCHE, Praxis worked directly with CBOs to build organizational capacity, support policy advocacy campaigns (resulting in 72 policy wins that increased access to nutritious and affordable food and safe places to play), and develop a national network of over 200 grassroots social justice CBOs (Grills et al., 2014). Phase 3 sought to leverage these networks and relationships to gain insights about best practices and emerging strategies used by organizers to advance health equity efforts. 
CCHE network members are working on any number of social justice issues including fair housing, environmental justice, and police accountability, all of which ultimately advance health equity. CBOs often operate with limited resources (i.e., small budgets, reliance on part-time staff or volunteers, etc.) and because campaigns and advocacy work often focus on the local context, community organizing work can be isolating. The landscape analysis presented an opportunity to learn from a wide range of organizers, produce knowledge, and share resources that could be distributed across the CCHE network.

\section{Process}

Under the leadership of Praxis, we developed a five-step process to analyze the landscape of childhood health inequities. Consistent with our CBPR approach, project partners (described below) were involved at every stage of the process.

\section{Step 1: Identify Shared Meaning}

In coordination with our project partners, Praxis and the CCHE Collaborative (technical assistance providers and other leaders representing national and local community organizing groups affiliated with CCHE since Phase 1), we identified three primary functions of the landscape analysis process: 1) understand the environment and health of African American, American Indian, Asian and Pacific Islander, and Latino children in underresourced and underserved communities; 2) uplift the current work of grassroots $\mathrm{CBO}$, including programs, services, organizing activities, and social justice campaigns; and 3) identify key organizing strategies (or "communitydefined best practices") used by grassroots CBOs to improve childhood health, including strategies that are not necessarily labeled as public health strategies. These initial conversations took place primarily via phone and teleconference among key project partners (i.e., PARC@LMU, Praxis, and CCHE Collaborative members).

\section{Step 2: Develop Sampling Strategy and Instruments}

Once the project partners agreed to the scope and purpose of the landscape analysis, we were ready to begin developing the methodology and analytic plan. A combined strategy was used to ensure that both rigorous qualitative methods and a community organizing focus guided the instrument development, codebook creation, and data analysis.

\section{Sampling Strategy}

To ensure a representative sample of diverse CBOs (in terms of geographic location and ethnic/racial communities served) the key informant groups were stratified by region (Midwest, Northeast, South, Southwest, and West) and ethnic/racial constituency group (African American, Asian and Pacific Islander, Latino, Indian Country, and Mixed Constituency). Our project partners assisted with developing a list of potential key informants on a shared Google spreadsheet, which allowed individuals to add suggestions, pose questions to each other, and edit the list in real time. The initial sampling 
Table 1. Final sample by ethnic/racial constituency and geographic location $(\mathrm{N}=45)$

\begin{tabular}{|c|c|c|c|c|c|c|}
\hline & African American & API & Latino & Indian Country & Mixed & Total \\
\hline Midwest & 1 & 2 & 1 & 4 & 1 & 9 \\
\hline Northeast & 2 & 6 & 1 & 0 & 2 & 11 \\
\hline South & 4 & 0 & 0 & 0 & 1 & 5 \\
\hline Southwest & 0 & 0 & 5 & 1 & 0 & 6 \\
\hline West & 2 & 3 & 2 & 1 & 6 & 14 \\
\hline Total & 9 & 11 & 9 & 6 & 10 & 45 \\
\hline
\end{tabular}

pool was drawn from existing network members (from CCHE Phases 1 and 2) and CCHE Collaborative contacts (these included organizations that were not part of the CCHE network previously, but that were engaged in community organizing work that impacted children).

We revised recruitment strategies with input from our project partners and reduced the list of potential key informants from 34 to 20 groups using the following criteria: 1) CBOs representing desired geographic regions and ethnic/racial constituencies; 2) CBOs who knew their communities well and were also focused on improving the lives of children using community-defined practices; and 3) those with whom Praxis and/or CCHE Collaborative members had an existing relationship. Organizations selected for the landscape analysis were engaged in community organizing that may or may not have an explicit focus on childhood health. For example, many CBOs in our sample do not define themselves as health organizations (i.e., focus areas include immigrant rights, economic justice, etc.), but nevertheless, their organizing work impacts community, and particularly children's, health. Social determinants of health (e.g., racism, poverty, etc.) are central to any public health strategy, especially one aiming to reduce inequity, and can best be discerned through the type of community engagement at which organizers excel.

Through an iterative process, we dropped and added CBOs from the list depending on the interest and availability of key informants. We exceeded our target of 20 interviews by December 2016, with a total sample of 23 organizations. Snowball sampling was used to generate a second sampling pool with 64 new organizations identified by key informants from the first round of interviews. Again, with input from CCHE Collaborative members, we reduced the list of potential key informants from 64 to 26 groups using the same criteria from the first round of interviews. As a result, 49 groups were dropped from the initial list and 11 groups were added for a target goal of 26 interviews. Four groups were not available to complete the interview before the end date in September 2017, resulting in a total sample of 22 interviews. The final sample across two rounds of interviews included 45 key informants representing grassroots CBOs across the country. See Table 1 for ethnic/racial constituency and geographic representation breakdown for both rounds of interviews. 


\section{Create Instruments}

We designed the key informant phone interview protocol in May 2016 with input from project partners. The interview protocol included a statement about confidentiality and the purpose of the landscape analysis, a brief quantitative assessment of organizational capacity needs and strengths (five minutes), and a semi-structured interview ( 45 to 60 minutes). We found that interviewees were more likely to respond to the organizational capacity survey while we had them on the phone for the interview, rather than try to get them to complete it on their own before or after the phone call. The interviewer read the brief survey questions aloud and recorded their answers in Qualtrics (online survey tool) on behalf of the respondent, and then transitioned to the semi-structured interview questions. The call was recorded on Zoom and the interviewer was trained to take written notes of high-level themes and key words or phrases. In total, the phone call took about one hour and covered the following topics: 1) Social Conditions, Barriers, and Impacts: features of the social and physical environment that inhibit healthy living and impact childhood health; 2) Current Work of the Organization: successful campaigns, efforts and programs to improve health, wellness, or safety in the community; and 3) Best Practices: strategies used by the organization to make a change that matters.

Consistent with our CBPR process, we piloted the interview protocol in June 2016 with four social justice organizations (two Los Angeles-based CBOs and two national non-profits from Indian Country). The protocol underwent substantive changes incorporating observations from the pilot interviews and feedback from our project partners. A slightly modified protocol was developed in consultation with Native Organizers Alliance, a longtime CCHE partner and technical assistance provider, for use with Indian Country constituency members. We finalized the interview protocol, including administration guidelines and questions, in July 2016 and began interviews in August 2016.

\section{Step 3: Data Collection}

The landscape analysis data included interviews with key informants and a targeted review of various information sources about each organization and their respective communities (i.e., ethnic/racial and geographic region). PARC@LMU, Praxis, and the CCHE Collaborative comprised the interview team, who jointly conducted 45 key informant interviews over 13 months. Given PARC@LMU's expertise in mixed methods data collection, we trained the team of eight interviewers on how to administer the organizational capacity survey via Qualtrics and conduct the semi-structured interviews. PARC@LMU was also responsible for scheduling and recording the phone interviews. Both PARC@LMU and Praxis transcribed the interviews for analysis. Interviews were conducted in two stages, as described above in the sample procedures section. 
Concurrently, PARC@LMU researchers reviewed documents about the CCHE communities to shed light on the health environment and social contexts that impact children's health. Specifically, our team pulled information from government sources (e.g., school and education outcomes, health dashboards, crime data, etc.), grey literature including policy papers and reports published by $\mathrm{CBO}$ s or foundations, $\mathrm{CBO}$ websites and social media, and other media (e.g., news coverage about $\mathrm{CBO}$ campaigns). These data-gathering activities helped to provide localized context and relevant information to interpret the key informant interviews through the appropriate lens and to develop written snapshot profiles of each organization (described in greater detail below).

\section{Step 4: Data Analysis}

We used an inductive approach to identify specific concepts in the interview transcripts that explain and give meaning to community-defined best practices within a specific cultural and community context as it relates to childhood health inequities. There were two phases in the coding process: 1 ) develop a first draft of the codebook based on a sample of interviews and 2) analyze all 45 interviews using a refined codebook.

\section{Phase 1 Coding}

The coding team (initially comprised of three qualitative researchers) was both interdisciplinary and multi-ethnic, which is integral to our process as it brought different perspectives and insights to the analysis. Two of the three coders were also involved in the documents review of health environment and social context information described above. The three-person team read an initial set of ten randomly selected interviews to develop conceptual categories ("codes") to fit the interview data. This included use of open-coding procedures and comparison of codes among the coders to verify their descriptive content and confirm that they were grounded in the data. The coders also used analytic memo writing to facilitate their individual analysis and interpretation of the data, which in turn informed the iterative and collaborative coding process. Through a consensus coding process, the codes were collapsed and organized in a codebook consisting of three large themes, 10 codes, and 20 sub-codes.

\section{Phase 2 Coding}

At this point a community organizing consultant joined the coding team to further refine the codebook and confirm that the codes aligned with social justice organizing principles and were reflective of community organizing philosophies and strategies. The organizing expert, while not part of the CCHE Network, had over 10 years of experience as a community organizer and held a leadership position at a social justice CBO. Their insights at both the organizing and operational levels helped us to add nuance and precision to the codebook and ensure we included appropriate indicators of social justice 
community organizing concepts. Using the refined codebook, the four-person team then recoded the initial ten interviews and the community organizing expert coded the remaining thirty-five interviews.

In August 2017, preliminary analyses were presented to the CCHE Strategic Advisory Committee (consisting of childcare providers and educators, community organizers, and childhood health advocates) to vet our findings. The providers in the room affirmed many of our findings related to current social conditions and barriers to childhood health in their respective communities. For example, key informants reported concerns with school discipline practices towards youth of color as young as five years old. Community organizers from California and Colorado validated this finding and elaborated on the problem of "school push-out" occurring in their communities, and how they are organizing parents and educators to end these harmful policies. The themes from the codebook capture the realities of conducting health equity and social change work in underrepresented communities.

\section{Step 5: Data Dissemination}

One goal of the landscape analysis process was to inform the social justice and organizing community about relevant work that is happening in other parts of the country in order to broaden the network and facilitate coalition building. This meant identifying ways to share findings beyond traditional research forums (i.e., conference presentations, academic journals), so that insights gained from the interviews could reach our intended audience. In consultation with project partners, and in accordance with the CBPR principle of shared decision-making regarding dissemination of findings, we developed 45 snapshot profiles - brief one- to two-page handouts tailored to each organization's specific community and cultural context that can be easily shared and utilized by practitioners. In addition, Praxis disseminated the snapshots via their website (https://www.thepraxisproject.org/) to raise awareness and understanding of community organizing efforts vis-à-vis social determinants of health.

The snapshots highlight the interplay between children's health, local community conditions and critical issues, organizational wellness and safety efforts, and organizational best practices and accomplishments. In addition to the key informant interviews themselves, several other data sources were used to provide relevant contextual information including regional health statistics (e.g., local health department), administrative data (e.g., census), organizational websites and social media pages, and other public documents and records (e.g., health reports, journal articles, news articles). The snapshots contain brief descriptions of the following: 1) organizational overview including their history, focus areas, and geographic location; 2) mission and/or vision statement; 3) constituencies served; 4) local conditions or barriers in the social and physical environment impacting children's health in the target community; 5) prevalence of key childhood health indicators, such as childhood obesity, low birth weight, physical activity, and asthma affecting 
children in the target community; 6) allies or partnerships with other community groups engaged in innovative work around childhood health; 7) ongoing health and wellness efforts, programs, or campaigns at the organization; 8) accomplishments (e.g., policy wins) related to community wellness and safety; 9) community-defined "best practices" or strategies used by the $\mathrm{CBO}$ to create long-term community change; and 10) contact information, including website and social media. Direct interview quotes are included to nuance and augment the data. Each snapshot was verified and approved by the key informant before being shared with Praxis, who designed the visual layout. The final snapshots were then shared back with the CBOs for their own use as promotional material.

\section{Findings}

The landscape analysis revealed challenges familiar to low-income communities of color including limited employment opportunities, lack of affordable housing, unsafe neighborhood conditions, and poor education resources. And although the social and environmental conditions are culturally and contextually distinct within each community, they are also intertwined, complex, and multi-faceted. This has resulted in profound collective and lasting social consequences on the health of children, families, and communities.

Our findings confirm the important role that community-based organizations play in supporting childhood health, regardless of whether an organization's stated focus is children and/or health. For example, during their key informant interview, an organizer for housing justice and tenant rights described how the stress and fear experienced by families dealing with landlord harassment and neglect trickles down from the parents to the children, contributing to emotional and physical health issues among young children (e.g., anxiety, trouble sleeping, stomach aches, etc.). Organizers in this setting provide tangible and emotional support to families facing eviction.

An unexpected finding was the extent to which community organizing $\mathrm{CBO}$ in our sample serve as a part of the social safety net for poor and working families by providing and/or linking their constituents to support services and resources (given their diverse and immense individual and community needs). One important implication of these findings is to consider holistic community organizing approaches that account for culture and context when attempting to improve childhood health. For example, the community organizers we spoke with identified innovative solutions to these significant challenges, including the practice of leveraging social services (e.g., child care, food pantries) and base-building activities (e.g., youth leadership development) to involve residents and young people in direct-action campaigns. By integrating strategies, organizers help build community power while simultaneously addressing the most pressing needs in the community. These findings challenge conventional funding mechanisms, which can reinforce silos rather than support the integrated way work happens on the ground. 


\section{Strengths and Limitations of the Landscape Analysis Approach}

A strength of the landscape analysis process was the stratified sampling procedures used to generate a diverse sample of key informants representing various ethnic/racial constituencies and geographic regions. The key informant interviews with community-organizing leaders illustrated the realities of social change efforts in underrepresented communities and provided concrete strategies that can be shared with other organizers.

Another strength is that the method was tailored to encompass CBPR principles and practices including trust and relationship building, collaboration, and centering community knowledge (Minkler et al., 2019; Wallerstein \& Duran, 2010). First, the deep and long-standing relationships among Praxis, the CCHE Collaborative members, and the key informants was crucial to our sampling and data collection strategies. Not only was insider knowledge necessary for us to become aware of certain CBOs, especially newer organizations, but the well-regarded reputations of Praxis and CCHE within social justice organizing circles contributed to organizers' willingness to talk with us. In essence, they "vouched" for us as researchers who were committed to social justice and could be trusted to honor the data and respect the communities that shared their stories. Second, the CCHE Collaborative was essential in the development and refinement of the data collection tools-their familiarity with the community-organizing context and subject-matter expertise ensured relevant and appropriate questions were included in our interview protocol, which yielded rich data and new insights.

A common challenge cited by practitioners of CBPR is the time-consuming and labor-intensive nature of collaborative, community-based work (Wallerstein \& Duran, 2006). We found this to be the case with the landscape analysis process as well. The development of our sampling strategy and instruments, data collection, and data analysis were all collaborative processes that took place over several months to ensure that we integrated feedback from project partners. Our methodology relied on in-depth interviews, which are time-consuming and create a significant barrier to data collection. In fact, we encountered challenges in making contact and scheduling interviews with some key informants who did not have time to be interviewed or simply were not interested in participating. When asked for names of potential key informants as part of our snowball sampling procedure, one Executive Director told the interviewer he doubted any of the names he provided would make time for an interview because there is too much day-to-day organizing work. A serious consideration for community-engaged researchers is how to lessen the burden on already overworked leaders in social justice community organizing movements. We attempted to mitigate this barrier by providing a tangible resource, the organizational snapshot profile, as an incentive for participation. Data analysis was also labor intensive as our multidisciplinary data analysis team went through several rounds of coding and revision. 
Additionally, resource constraints limited the number of expert advisors we were able to include on our team. Although youth were involved at the level of the actual organizations (i.e., as members and leaders) included in the landscape analysis, they were not directly involved in the instrument development and data analysis process. Similarly, we were not able to include more than one community-organizing expert in the data analysis process. That said, the community-organizing expert played a significant role in revising our codebook and framing our findings given their knowledge of processes, constructs, and trends in the field. Specifically, they directed attention to the "integrated strategies" that $\mathrm{CBO}$ s are using (i.e., combining direct service and direct action) as a noteworthy pattern in our data that has implications for the broader field.

\section{Conclusion}

Participatory methods like landscape analysis offer a useful tool for applied public health researchers to produce big-picture assessments that can aid in identifying trends and promising practices across a large sample, without sacrificing the richness of individual-level qualitative data. Participatory research increases the ability to authentically capture the factors affecting health and health inequities in communities of color and marginalized communities by revealing the drivers of social determinants of health that are often overlooked in traditional research. The findings underscore the importance of a large, well-established network like CCHE that serves as a critical hub for connecting seemingly dissimilar organizations and supporting local, community-based organizing and advocacy to directly confront racial injustice and health inequities. For close to a decade, the CCHE project has contributed to the general knowledge about the social determinants of health nuanced to community context, culture, and place and is helping to shift the conventional wisdom about what constitutes "public health" work to be more inclusive of grassroots community-organizing strategies and activities (Douglas et al., 2016; Grills et al., 2014; Subica et al., 2016).

Grassroots CBOs play a critical role in advancing health equity through supporting individual and collective agency among historically disenfranchised communities of color (e.g., building strategic alliances with other like-minded groups to leverage power and shape decision making) and fighting for systems and policy changes using a racial justice and health equity framework that takes into account the socio-cultural-political context of their local communities. Community organizers use landscape analysis to deepen understanding of the structural causes of social conditions and to mobilize and engage those most affected in the definition of issues and construction of solutions (i.e., policy and systems change campaigns) to shift power and transform systems. Therefore, any public health strategy that seeks to address root causes and promote sustainable changes that improve health must work with, and through, these grassroots CBOs. Closing the gaps in health inequities to achieve social justice and health equity requires public health to pursue nontraditional methodologies like community organizing. 
This is an open-access article distributed under the terms of the Creative Commons Attribution 4.0

International License (CCBY-4.0). View this license's legal deed at http://creativecommons.org/licenses/ by/4.0 and legal code at http://creativecommons.org/licenses/by/4.0/legalcode for more information. 


\section{References}

Blumenshine, P., Egerter, S., Barclay, C. J., Cubbin, C., \& Braveman, P. A. (2010). Socioeconomic disparities in adverse birth outcomes: A systematic review. American Journal of Preventive Medicine, 39(3), 263-272. https://doi.org/10.1016/j.amepre.2010.05.012

Braveman, P., \& Barclay, C. (2009). Health disparities beginning in childhood: A life-course perspective. Pediatrics, 124(Supplement 3), S163-S175. https://doi.org/10.1542/peds.2009-1100d

Braveman, P., Egerter, S., \& Williams, D. R. (2011). The social determinants of health: Coming of age. Annual Review of Public Health, 32(1), 381-398. https://doi.org/10.1146/annurevpublhealth-031210-101218

Castellanos, P., \& Pateriya, D. (2003). Power tools: A manual for organizations fighting for justice. Strategic Concepts in Organizing and Policy Education (SCOPE). Los Angeles.

Christens, B. D., \& Speer, P. W. (2015). Community organizing: Practice, research, and policy implications. Social Issues and Policy Review, 9(1), 193-222. https://doi.org/10.1111/sipr.12014

Cohen, A. K., Lopez, A., Malloy, N., \& Morello-Frosch, R. (2016). Surveying for environmental health justice: Community organizing applications of community-based participatory research. Environmental Justice, 9(5), 129-136. https://doi.org/10.1089/env.2016.0008

Douglas, J. A., Grills, C. T., Villanueva, S., \& Subica, A. M. (2016). Empowerment praxis: Community organizing to redress systemic health disparities. American Journal of Community Psychology, 58(3-4), 488-498. https://doi.org/10.1002/ajcp.12101

Fisher, R., DeFilippis, J., \& Shragge, E. (2018). Contested Community: A Selected and Critical History of Community Organizing. In R. A. Cnaan \& C. Milofsky (Eds.), Handbook of Community Movements and Local Organizations in the 21st Century (pp. 281-297). Springer International Publishing. https://doi.org/10.1007/978-3-319-77416-9_17

Flores, G. (2010). Technical report: Racial and ethnic disparities in the health and health care of children. Pediatrics, 125(4), e979-e1020. https://doi.org/10.1542/peds.2010-0188

Graham, P., Evitts, T., \& Thomas-MacLean, R. (2008). Environmental scans: How useful are they for primary care research? Canadian Family Physician, 54(7), 1022-1023.

GrantCraft. (2012). Scanning the landscape 2.0: Finding out what's going on in your field. http://grantcraft.org/wp-content/uploads/sites/2/2018/12/scan20.pdf

Grills, C., Villanueva, S., Subica, A. M., \& Douglas, J. A. (2014). Communities creating healthy environments: Improving access to healthy foods and safe places to play in communities of color. Preventive Medicine, 69(Suppl. 1), S117-S119. https://doi.org/10.1016/j.ypmed.2014.10.026

Guerrero, A. D., Mao, C., Fuller, B., Bridges, M., Franke, T., \& Kuo, A. A. (2016). Racial and ethnic disparities in early childhood obesity: Growth trajectories in body mass index. Journal of Racial and Ethnic Health Disparities, 3(1), 129-137. https://doi.org/10.1007/s40615-015-0122-y

Gutiérrez, L. M., \& Lewis, E. A. (2012). Education, participation and capacity building in community organizing with women of color. In M. Minkler (Ed.), Community Organizing and Community Building for Health and Welfare (3rd ed., pp. 215-228). Rutgers University Press.

Israel, B. A., Parker, E. A., Rowe, Z., Salvatore, A., Minkler, M., López, J., Butz, A., Mosley, A., Coates, L., Lambert, G., Potito, P. A., Brenner, B., Rivera, M., Romero, H., Thompson, B., Coronado, G., \& Halstead, S. (2005). Community-based participatory research: Lessons learned from the centers for children's environmental health and disease prevention research. Environmental Health Perspectives, 113(10), 1463-1471. https://doi.org/10.1289/ehp.7675

Israel, B. A., Schulz, A. J., Parker, E. A., \& Becker, A. B. (1998). Review of community-based research: Assessing partnership approaches to improve public health. Annual Review of Public Health, 19(1), 173-202. https://doi.org/10.1146/annurev.publhealth.19.1.173 
Israel, B. A., Schulz, A. J., Parker, E. A., Becker, A. B., Allen, A. J., \& Guzman, J. R. (2003). Critical issues in developing and following community-based participatory research principles. In M. Minkler \& N. Wallerstein (Eds.), Community-Based Participatory Research for Health (pp.56-73 (pp. 56-73). Jossey-Bass.

Larson, K., Russ, S. A., Crall, J. J., \& Halfon, N. (2008). Influence of multiple social risks on children's health. Pediatrics, 121(2), 337-344. https://doi.org/10.1542/peds.2007-0447

Learning for Action. (2017). Developing systems to serve the mental health needs of children 0-5 in San Mateo County: A landscape scan. First 5 San Mateo County. https://first5sanmateo.org/wpcontent/uploads/2017/11/F5-SMC-ECMH-Landscape-Scan_11-15-17.pdf

Mehta, N. K., Lee, H., \& Ylitalo, K. R. (2013). Child health in the United States: Recent trends in racial/ethnic disparities. Social Science E̊ Medicine, 95, 6-15. https://doi.org/10.1016/ j.socscimed.2012.09.011

Minkler, Meredith, Rebanal, R. D., Pearce, R., \& Acosta, M. (2019). Growing equity and health equity in perilous times: Lessons from community organizers. Health Education $\sigma^{\circ}$ Behavior, 46(1_suppl), 9S-18S. https://doi.org/10.1177/1090198119852995

Ogden, C. L., Carroll, M. D., Kit, B. K., \& Flegal, K. M. (2012). Prevalence of obesity and trends in body mass index among US children and adolescents, 1999-2010. JAMA, 307(5), 483-490. https://doi.org/10.1001/jama.2012.40

Pastor, M., Ito, J., \& Rosner, R. (2011). Transactions, Transformations, Translations: Metrics that matter for building, scaling, and funding social movements. USC Dornsife Program for Environmental and Regional Equity. https://dornsife.usc.edu/assets/sites/242/docs/ transactions_transformations_translations_web.pdf

Pastor, M., Terriquez, V., \& Lin, M. (2018). How community organizing promotes health equity, and how health equity affects organizing. Health Affairs, 37(3), 358-363. https://doi.org/10.1377/ hlthaff.2017.1285

Rowel, R., Moore, N. D., Nowrojee, S., Memiah, P., \& Bronner, Y. (2005). The utility of the environmental scan for public health practice: Lessons from an urban program to increase cancer screening. Journal of the National Medical Association, 97(4), 527-534.

Sanders-Phillips, K., Settles-Reaves, B., Walker, D., \& Brownlow, J. (2009). Social inequality and racial discrimination: Risk factors for health disparities in children of color. American Academy of Pediatrics, 124 (Supplement 3), S176-S186. https://doi.org/10.1542/peds.2009-1100e

Sherry, C. (2013). Fast forward to greater impact: The power of field scans. National Center for Family Philanthropy. https://www.ncfp.org/knowledge/fast-forward-to-greater-impact-the-power-of-field$\underline{\text { scans/ }}$

Speer, P. W., Ontkush, M., Schmitt, B., Raman, P., Jackson, C., Rengert, K. M., \& Peterson, N. A. (2003). The intentional exercise of power: Community organizing in Camden, New Jersey. Journal of Community $\Xi^{2}$ Applied Social Psychology, 13(5), 399-408. https://doi.org/10.1002/casp.745

Subica, A. M., Grills, C. T., Douglas, J. A., \& Villanueva, S. (2016). Communities of color creating healthy environments to combat childhood obesity. American Journal of Public Health, 106(1), 79-86. https://doi.org/10.2105/ajph.2015.302887

Wallerstein, N., \& Duran, B. (2006). Using community-based participatory research to address health disparities. Health Promotion Practice, 7(3), 312-323. https://doi.org/10.1177/1524839906289376

Wallerstein, N., \& Duran, B. (2010). Community-based participatory research contributions to intervention research: The intersection of science and practice to improve health equity. American Journal of Public Health, 100(S1), S40-S46. https://doi.org/10.2105/ajph.2009.184036 
Wilburn, A., Vanderpool, R. C., \& Knight, J. R. (2016). Environmental Scanning as a Public Health Tool: Kentucky's Human Papillomavirus Vaccination Project. Preventing Chronic Disease, 13, E109. https://doi.org/10.5888/pcd13.160165 